\title{
Bankrisiko und \\ Risikosteuerung mit Derivaten
}

\author{
UDO BROLL* \\ Universität des Saarlandes \\ Peter Welzel \\ Universität Augsburg
}

Juli 2002

In einem einfach gehaltenen Bankmodell untersucht der Beitrag die ökonomischen Wirkungen des Einsatzes von Derivaten für marktgängige und nicht marktgängige Risiken. Wir betrachten das klassische Kreditund Einlagengeschäft einer kompetitiven Bank. Sind Kreditausfall- und Zinsänderungsrisiko marktgängig, ergeben sich einfache Entscheidungsregeln. Für die Kredit- und Einlagenpolitik sind dann ausschließlich Marktund Kostengrößen relevant. Treten jedoch nicht handelbare Risiken auf, dann sind neben den Marktdaten der Grad der Risikoaversion, die Verteilung und die finanzielle Ausstattung der Bank bei der Bestimmung des optimalen Asset Liability Managements zu beachten. Der Beitrag unterstreicht die ökonomische Bedeutung von derivaten Märkten und des Einsatzes von Finanzderivaten zur Risikosteuerung in Banken.

* Korrespondenz: Udo Broll, Universität des Saarlandes, Fachbereich 1.2 (Volkswirtschaftslehre), Lehrstuhl für Internationale Wirtschaftsbeziehungen und Europäische Wirtschaft (Lehrstuhlvertretung), D-66041 Saarbrücken, Fax: 0681-302-4390, E-mail: u.broll@mx.uni-saarland.de 


\section{Bankrisiko und Risikosteuerung mit Derivaten}

PD Dr. Udo Broll, Saarbrücken und Prof. Dr. Peter Welzel, Augsburg

In einem einfach gehaltenen Bankmodell untersucht der Beitrag die ökonomischen Wirkungen des Einsatzes von Derivaten für marktgängige und nicht marktgängige Risiken. Wir betrachten das klassische Kreditund Einlagengeschäft einer kompetitiven Bank. Sind Kreditausfall- und Zinsänderungsrisiko marktgängig, ergeben sich einfache Entscheidungsregeln. Für die Kredit- und Einlagenpolitik sind ausschließlich Markt- und Kostengrößen relevant. Treten jedoch nicht handelbare Risiken auf, dann sind neben den Marktdaten der Grad der Risikoaversion, die Verteilung und die finanzielle Ausstattung der Bank bei der Bestimmung des optimalen Asset Liability Managements zu beachten. Der Beitrag unterstreicht die ökonomische Bedeutung von derivaten Märkten und des Einsatzes von Derivaten zur Risikosteuerung in Banken.

\section{Einführung}

Als Finanzintermediäre erfüllen Banken Transformationsfunktionen. Sie bilden im traditionellen Einlagen- und Kreditgeschäft die Brücke zwischen Kapitalgebern und Kapitalnehmern mit unterschiedlichen Vorstellungen über Anlage- bzw. Kreditbeträge und -fristen. In diesem traditionellen Kerngeschäft der Banken entstehen Risiken - insbesondere ein Kredit- und ein Refinanzierungsrisiko (für eine Übersicht zu Risiken des Bankgeschäfts vgl. Zink, 2001). Risikomanagement stellt deshalb eine der Hauptaufgaben des Bankmanagements dar, deren Bedeutung aufgrund höherer Volatilitäten, verschärften Wettbewerbsdrucks und institutioneller Änderungen (Bankenregulierung nach Basel I, Basel II) zugenommen hat. Erweiterte Spielräume ergeben sich für das Risikomanagement in Banken durch die Entwicklung und Bereitstellung neuer derivativer Finanzkontrakte (vgl. Deutsche Bundesbank, 1993, 2002, Hartmann-Wendels et al., 2000, Broll/Jaenicke, 2000).

Als Ergebnisvariable des Risikomanagements der Bank kommen der Marktwert des Zahlungsstroms oder der Zahlungsstrom selbst in Frage. Die Zielsetzung des Risikomanagements wird letztendlich von den Interessen der Kapitalgeber bestimmt (Pritsch/Hommel, 1997). Unser Beitrag untersucht auf der Zahlungsstromebene die ökonomischen Wirkungen des Einsatzes von Finanzderivaten zur Absicherung von Kredit- und Refinanzierungsrisiko. Wir verwenden das Grundmodell des industrieökonomischen Ansatzes der Banktheorie (vgl. Freixas/Rochet, 1998, Kap. 3; zur Integration von Unsicherheit vgl. Wahl/Broll, 2000, Wahl/Broll, 2001, Pausch/Welzel, 2002) und betrachten eine Bank bei vollkommener Konkurrenz.

Das Refinanzierungsrisiko wird als handelbar unterstellt. Wir begründen diese Annahme mit den institutionellen Gegebenheiten des Interbanken- und des Geld- 
markts: Standardisierung, hohe Liquidität, Qualität der Marktteilnehmer. Das Risiko aus der Kreditrückzahlung hingegen galt bislang als in der Regel nicht marktgängig, da ein potentieller Käufer eines Kredits oder Kreditportfolios gravierende Informationsnachteile gegenüber der ursprünglich Kredit gewährenden Bank aufweist (vgl. Hartmann-Wendels et al., 2000, S. 766f.). Bei näherer Betrachtung stellt sich das mit einem Kredit verbundene Risiko in Analogie zu einer Aktie als in einen systematischen Teil (Marktrisiko) und einen unsystematischen Teil (idiosynkratisches Risiko) zerlegbar dar. Das Marktrisiko, das durch wenige makroökonomische Faktoren erklärt werden kann (vgl. Wilson, 1998), bestimmt einen Großteil des Kreditrisikos und ist als handelbar anzusehen. Das idiosynkratische Risiko des einzelnen Kredits hingegen sollte im Idealfall durch die Bildung eines Kreditportfolios von der Bank selbst beseitigt werden. Wenn Banken dazu — z.B. aufgrund des Regionalprinzips bei Sparkassen und Genossenschaftsbanken oder durch Bindungen an einzelne Sektoren der Wirtschaft — nicht in der Lage sind, dann wären sie auch für diesen (kleineren) Teil des Kreditrisikos an einer Handelbarkeit interessiert. Die erwähnten Informationsprobleme erschweren oder verhindern aber den Handel mit einem solchen spezifischen Risiko. In jüngerer Zeit haben sich Märkte für Kreditderivate rasant entwickelt (vgl. Burghof et al., 2000). Wir analysieren deshalb zunächst den Fall ohne Handelbarkeit des Kreditrisikos und führen in einem zweiten Schritt ein Kreditderivat zur perfekten Handelbarkeit ein.

Folgende Fragen stehen im Mittelpunkt des vorliegenden Beitrages. Wie beeinflusst das Refinanzierungsrisiko die optimale Kredit- und Einlagenpolitik der Bank, wenn Finanzderivate zur Risikosteuerung genutzt werden können? Die Analyse zeigt: Sind alle Risiken marktgängig, ergeben sich einfache Entscheidungsregeln für die Festlegung der optimalen Bankpolitik. Neben den operativen Kosten sind allein Marktdaten für die Entscheidungen des Bankmanagements relevant. Sind jedoch nicht marktgängige Risiken, in unserem Fall Kreditausfallrisiken, zu berücksichtigen, dann sind die Entscheidungsregeln erheblich zu relativieren. Die Geschäftspolitik basiert nicht mehr allein auf Marktdaten und kann auch nicht mehr präferenzfrei formuliert werden. Es spielen Renditeerwartungen, der Grad der Risikoaversion des Bankmanagements, die Korrelation zwischen Kredit- und Marktzinssatz und die finanzielle Anfangsausstattung der Bank eine zentrale Rolle bei der Bestimmung der optimalen Kredit- und Einlagenpolitik.

Ziel unseres Beitrages ist es, den ökonomischen Zusammenhang zwischen dem Risikomanagement von marktgängigen und nicht marktgängigen Risiken für die Kreditund Einlagenpolitik eines Finanzintermediärs aufzuzeigen. Die Analyse zeigt, dass Finanzderivate nicht nur der Risikoreduktion dienen, sondern auch auf die simultane Entscheidung der Kreditvergabe und die Hereinnahme von Einlagen zurückwirken.

Der Beitrag ist wie folgt aufgebaut. In Abschnitt 2 wird ein einfach gehaltenes Modell einer Bank vorgestellt. Die Kreditrückzahlung und die Kosten der Refinanzierung sind zum Planungszeitpunkt unsicher. Es entsteht ein Endvermögensrisiko für die Bank, da der Gewinn stochastisch ist. Das Bankmanagement ist risikoavers. Neben der Kredit- und Einlagenpolitik ist das Kontraktvolumen von Zinsfutures in Ab- 
schnitt 3 eine Entscheidungsvariable der Bankmanager. In Abschnitt 4 führen wir Kreditderivate ein und untersuchen ihre Auswirkungen auf die Geschäftspolitik der Bank. Abschnitt 5 faßt die wichtigsten Ergebnisse zusammen.

\section{Das Modell}

Grundlage ist ein einfaches Bankmodell in der Tradition des industrieökonomischen Ansatzes der Bankentheorie. Es besteht ein Endvermögensrisiko durch Unsicherheit bei der Rückzahlung aus der Kreditvergabe und bei den Refinanzierungskosten der Bank. Die Bank reicht zu Beginn der Periode ein Kreditvolumen $L$ aus und vereinbart eine Rückzahlung $L_{1}$ am Periodenende. Hieraus ergibt sich eine Effektivverzinsung $r_{L}=\left(L_{1}-L\right) / L$. Tatsächlich ist aber die Rückzahlung unsicher. Wir bezeichnen mit $\tilde{L}_{1}$ die auf dem Intervall $\left[0, L_{1}\right]$ verteilte Zufallsvariable der Kreditrückzahlung. Hieraus ergibt sich aus Sicht der Bank eine unsichere Effektivverzinsung $\tilde{r}_{L}=\left(\tilde{L}_{1}-L\right) / L$. Das Kreditvolumen $L$ ist neben dem Einlagevolumen $D$ die Entscheidungsvariable der Bank. Bei einem gegebenen Einlagenzins $r_{D}$ ist der Zinsaufwand aus dem Passivgeschäft $r_{D} D$. Die operativen Kosten der Bank sind durch die Kostenfunktion $C(L, D)$ mit positiven Grenzkosten beschrieben.

Bei einem gegebenen Eigenkapital $K_{0}$ der Bank ergibt sich ein Finanzierungsüberschuss $B \geq 0$ bzw. eine Finanzierungslücke $B<0$ anhand der Bankbilanzbedingung

$$
L+B=K_{0}+D .
$$

Im Umfang $B$ engagiert sich die Bank im Interbankenmarkt zu einem unsicheren Zinssatz $\tilde{r}$. Der unsichere Refinanzierungszins lässt sich intuitiv mit einer längeren Laufzeit des Kreditgeschäfts im Vergleich zum Refinanzierungsgeschäft erklären.

Mit einem Anfangsvermögen von Null und ohne Einsatz von Derivaten ergibt sich das unsichere Endvermögen $\tilde{W}_{O H}$ der Bank als Summe aus (unsicherer) Kreditverzinsung und (unsicherem) Zinserlös aus dem Interbankenmarkt abzüglich Zinsaufwand für Einlagen und operative Kosten:

$$
\tilde{W}_{O H}=\tilde{r}_{L} L+\tilde{r} B-r_{D} D-C(L, D) .
$$

Ein Zinsfuture im Umfang $X$ auf den Zins $\tilde{r}$ garantiert zum Periodenende die sichere Zahlung $r_{X} X$ im Austausch gegen die Realisierung von $\tilde{r} X$. Der Zinsfuture trägt aus der Sicht des Periodenbeginns den (unsicheren) Differenzgewinn bzw. - verlust $\left(r_{X}-\tilde{r}\right) X$ zum Endvermögen bei.

Ungeachtet des Variantenreichtums bei den Kreditderivaten der Realität (vgl. Hartmann-Wendels et al., 2000, S. 295ff.) konzentrieren wir uns auf den ökonomischen Kern dieser Finanzkontrakte. Es handelt sich auch hier um den Austausch einer unsicheren zukünftigen gegen eine sichere zukünftige Zahlung: Ein Kreditderivat im Umfang $Y$ auf den Effektivzins $\tilde{r}_{L}$ garantiert zum Periodenende die sichere Zahlung $r_{Y} Y$ im Austausch gegen die Realisierung von $\tilde{r}_{L} Y$. Das Kreditderivat trägt den (unsicheren) Differenzgewinn bzw. -verlust $\left(r_{Y}-\tilde{r}_{L}\right) Y$ zum Endvermögen bei. 
Bei Nutzung beider Derivate sind die Ergebnisbeiträge der Hedging-Operationen im Endvermögen zu berücksichtigen. Die Definition (2) wird ergänzt zu

$$
\tilde{W}=\tilde{W}_{O H}+\left(r_{X}-\tilde{r}\right) X+\left(r_{Y}-\tilde{r}_{L}\right) Y .
$$

Zu maximieren ist der erwartete Nutzen des unsicheren Endvermögens $\tilde{W}$

$$
\max \mathrm{E}[U(\tilde{W})]
$$

mit dem Erwartungsoperator E und der Nutzenfunktion $U, U^{\prime}>0, U^{\prime \prime}<0$. Die Präferenzfunktion dient der Bewertung der Wahrscheinlichkeitsverteilung des Risiko behafteten Endvermögens. Die Risikoaversion des Bankmanagements wird durch die Konkavität von $U$ ausgedrückt.

In Abschnitt 3 gehen wir davon aus, dass der Bank nur Zinsfutures zur Absicherung zur Verfügung stehen. Sie entscheidet simultan über das Kreditvolumen $L$, das Einlagenvolumen $D$ und das Volumen von Zinsfutures $X$. Im darauf folgenden Abschnitt 4 wird die Analyse um Kreditderivate erweitert. Die Bank bestimmt dann gemeinsam mit den drei erwähnten Größen auch das Volumen an Kreditderivaten $Y$.

\section{Nicht handelbare Kreditrisiken}

\subsection{Optimale Kredit- und Einlagenpolitik}

Maximierung der Zielfunktion (4) unter Berücksichtigung der Bankbilanz (1) bezüglich der Entscheidungsvariablen $L, D$ und $X$ liefert die folgenden Bedingungen für die optimale Politik der Bank:

$$
\begin{aligned}
E\left[U^{\prime}\left(\tilde{W}^{*}\right)\left(\tilde{r}_{L}-\tilde{r}-C_{L}\right)\right] & =0, \\
E\left[U^{\prime}\left(\tilde{W}^{*}\right)\left(\tilde{r}-r_{D}-C_{D}\right)\right] & =0, \\
E\left[U^{\prime}\left(\tilde{W}^{*}\right)\left(r_{X}-\tilde{r}\right)\right] & =0,
\end{aligned}
$$

wobei $U^{\prime}$ den positiven Grenznutzen des Endvermögens $\tilde{W}^{*}$ und $C_{L}, C_{D}$ die Grenzkosten $C_{L}\left(L^{*}, D^{*}\right)$ bzw. $C_{D}\left(L^{*}, D^{*}\right)$ der Kredit- und Einlagenpolitik im Optimum darstellen. Die Bank agiert nur dann als Finanzintermediär, wenn die erwartete Zinsspanne im Kredit- bzw. Einlagengeschäft die operativen Grenzkosten des jeweiligen Geschäftsfelds übersteigt. Die weitere Analyse unterstellt, dass dies der Fall ist.

Wir fassen das erste Ergebnis wie folgt zusammen.

Aussage 1 (Optimale Kredit- und Einlagenpolitik) (a) Im Optimum der Bank werden Kredit- und Einlagenvolumen neben den Marktdaten und den operativen Grenzkosten vom Grad der Risikoaversion und den Erwartungen des Bankmanagements bestimmt. (b) Nur in einem Spezialfall, $C_{L D}=0$, wird das Einlagenvolumen $D^{*}$, 
nicht jedoch das Kreditvolumen $L^{*}$, ausschließlich von Marktdaten und Grenzkosten determiniert.

Beweis (a) Einsetzen von (7) in (5) und (6) liefert zwei Gleichungen, in denen $L^{*}$ und $D^{*}$ abhängig von allen Parametern des Entscheidungsproblems sind. (b) Die Verrechnung von (6) und (7) ergibt bei $C_{L D}=0$ als Entscheidungsregel $r_{X}-r_{D}=$ $C_{D}\left(L^{*}, D^{*}\right)$ für $D^{*}$.

q.e.d.

Die Hereinnahme einer zusätzlichen Einlage verursacht Zinsaufwand. Dem steht ein unsicherer Zinsertrag im Interbankenmarkt gegenüber, der über den Zinsfuture in einen sicheren Ertrag transformiert wird. Weiterhin lässt sich zeigen, dass im Opti$\operatorname{mum} \mathrm{E}\left(\tilde{r}_{L}\right)>r_{X}>r_{D}$ und $\mathrm{E}\left[U\left(\tilde{W}^{*}\right)\right] \geq \mathrm{E}\left[U\left(\tilde{W}_{O H}^{*}\right)\right]$ gelten müssen.

\subsection{Optimales Futures-Hedging}

Wir gehen zur Vereinfachung von einem unverzerrten Futures-Markt, $E(\tilde{r})=r_{X}$, aus. Das Ergebnis für ein optimales Futures-Hedging der Bank lässt sich wie folgt zusammenfassen:

Aussage 2 (Optimale Risikopolitik) (a) Sind Zinsänderungs- und Kreditausfallrisiko stochastisch unabhängig, dann ist das Refinanzierungsrisiko vollständig abzusichern, d.h. $X^{*}=B^{*}$. (b) Sind Zinsänderungs- und Kreditausfallrisiko positiv (negativ) korreliert, dann ist eine Überabsicherung (Unterabsicherung) $X^{*}>B^{*}\left(X^{*}<B^{*}\right)$ optimal.

Beweis Durch Anwendung der Rechenregel $E(x y)=E(x) E(y)+\operatorname{Cov}(x, y)$ und der Unverzerrtheit des Terminmarktes folgt aus (7)

$$
\operatorname{Cov}\left(U^{\prime}\left(\tilde{W}^{*}\right), \tilde{r}\right)=0,
$$

mit $\tilde{W}^{*}=\tilde{r}_{L} L^{*}+\tilde{r}\left(B^{*}-X^{*}\right)+$ konst.. Der konstante Term in (8) umfasst die sicheren Zahlungsströme $r_{D}^{*} D, C\left(L^{*}, D^{*}\right)$ und $r_{X} X^{*}$ aus (3). Gleichung (8) dient der Charakterisierung der optimalen Risikopolitik, da sie den ökonomischen Zusammenhang zwischen dem handelbaren Risiko und der Verstetigung des Endvermögens beschreibt. (a) Die Kovarianz zwischen Markzins $\tilde{r}$ und Grenznutzen ist bei stochastischer Unabhängigkeit zwischen Marktzins und Kreditzins dann und nur dann Null, wenn $X^{*}=B^{*}$ gilt. Dies entspricht einer Hedge-Rate von 100 Prozent. (b) Da die Kovarianz zwischen Grenznutzen und Marktzinssatz verschwindet, müssen sich (für beliebige Verteilungen) Grenznutzen und Marktzins entgegengerichtet entwickeln, um (8) erfüllen zu können. Entgegen Aussage (b) sei für eine positive Korrelation angenommen, dass eine Unterabsicherung $B^{*}<X^{*}$ optimal sei. Sind $\tilde{r}$ und $\tilde{r}_{L}$ positiv korreliert, dann führt dies zu einem Widerspruch, da die Unterabsicherung den Grenznutzen erhöht, während bei einem Anstieg von $r_{L}$ der Grenznutzen fällt. Folglich ist bei positiver Korrelation eine Überabsicherung optimal. Analog erfolgt die Beweisführung zur Unterabsicherung bei negativer Korrelation zwischen Marktzins und Kreditzins.

q.e.d. 
Wie lässt sich die optimale Risikopolitik, insbesondere die Unterabsicherung bzw. Überabsicherung, erklären? Im Rahmen des Risikomanagements mit Zinsfutures ist die optimale Hedge-Rate $X^{*} / B^{*}$ zu bestimmen. Enthält der Terminkurs keine Risikoprämie, $r_{X}=\mathrm{E}(\tilde{r})$, und sind Kreditausfallrisiko und Interbankenzins stochastisch unabhängig, dann beträgt die optimale Hedge-Rate 100 Prozent. Eine Abweichung von einem 100-Prozent-Hedge kann nur dann optimal sein, wenn eine Risikoprämie existiert oder eine Korrelation vorliegt. Sind Interbankenzins und Kreditzins negativ (positiv) korreliert, dann ist eine Unterabsicherung (Überabsicherung) optimal, denn die Risiken ergänzen sich in einer vorteilhaften (unvorteilhaften) Art und Weise. Ein Endvermögensrisiko bleibt jedoch bestehen. Die Leser sind eingeladen, die Argumentation dieses Abschnitts im einfachen Rahmen der $(\mu, \sigma)$-Entscheidungstheorie mit konstantem Grad der Risikoaversion zu überprüfen.

\section{Handelbare Kreditrisiken}

\subsection{Optimale Kredit- und Einlagenpolitik}

Wenn zusätzlich Kreditderivate zur Verfügung stehen, dann maximiert die Bank (4) unter Berücksichtigung der Bankbilanz (1) bezüglich der Entscheidungsvariablen $L$, $D, X$ und $Y$. Wir erhalten das folgende Ergebnis:

Aussage 3 (Trennbarkeitseigenschaft) (a) Wenn sich der Differenzgewinn bzw. verlust aus dem Kreditderivat exakt spiegelbildlich zum Risiko behafteten Cash flow aus dem Kreditgeschäft entwickelt (kein Basisrisiko), dann erfordert die optimale Kreditvergabe die Gleichheit der Intermediationsmarge $r_{Y}-r_{X}$ des Kreditgeschäfts und der operativen Grenzkosten der Kreditvergabe. (b) Wenn sich der Differenzgewinn bzw. - verlust aus dem Zinsfuture exakt spiegelbildlich zum Risiko behafteten Cash flow aus dem Interbankenmarkt entwickelt (kein Basisrisiko), dann erfordert die optimale Einlagenpolitik die Gleichheit der Intermediationsmarge $r_{X}-r_{D}$ des Einlagengeschäfts und der operativen Grenzkosten der Einlagen.

Beweis (a) Die Bedingung erster Ordnung für eine optimale Entscheidung bezüglich des Einsatzes $Y$ des Kreditderivats lautet

$$
\mathrm{E}\left[U^{\prime}\left(\tilde{W}^{*}\right)\left(r_{Y}-\tilde{r}_{L}\right)\right]=0
$$

Die Optimalitätsbedingungen für die Kreditvergabe (5), den Einsatz von Zinsfutures

(7) und den Einsatz von Kreditderivaten (9) führen auf

$$
r_{Y}-r_{X}=C_{L}\left(L^{*}, D^{*}\right) .
$$

(b) Verrechnung von (6) und (7) führen auf

$$
r_{X}-r_{D}=C_{D}\left(L^{*}, D^{*}\right) .
$$


Die Entscheidungsregeln (10) und (11) zeigen, dass weder der Grad der Risikoaversion des Bankmanagements, noch die Wahrscheinlichkeitsverteilung des Zinsänderungsrisikos und des Kreditausfallsrisikos, noch Renditeprognosen des Bankmanagements das optimale Kredit- und Einlagenvolumen beeinflussen, wenn es für die beiden Risiken zwei perfekte Hedging-Instrumente gibt. Es resultiert die aus der Mikroökonomik bei Sicherheit vertraute Forderung, Gleichheit von Grenzkosten und Grenzertrag herzustellen. Die Entscheidungen über Kredite und Einlagen können von den Risikoabsicherungsentscheidungen getrennt werden.

\subsection{Optimales Hedging}

Für das optimale Hedging liefert das Maximierungsproblem der Bank das folgende Ergebnis:

Aussage 4 (Full Double Hedge) (a) Bei unverzerrten Märkten für Zinsfutures und Kreditderivate $\left(r_{X}=\mathrm{E}(\tilde{r})\right.$ und $\left.r_{Y}=\mathrm{E}\left(\tilde{r}_{L}\right)\right)$ besteht die optimale Risikopolitik der Bank in einer Vollabsicherung beider Risiken. Dies führt zu einem sicheren Endvermögen. (b) Wenn einer der beiden oder beide Märkte für Hedging-Instrumente verzerrt ist bzw. sind, dann beeinflussen Korrelationen zwischen den Risiken und der Grad der Risikoaversion die optimale Risikopolitik.

Beweis (a) Sind beide Hedge-Instrumente unverzerrt, dann folgt aus der Optimalitätsbedingung (7) und (9) mit Einsetzen von (3) die vollständige Absicherung (full double hedge) $X^{*}=B^{*}$ und $Y^{*}=L^{*}$. Aufgrund der Vollabsicherung wird das Endvermögen $W^{*}$ zu einer sicheren Größe, da es im Optimum nur von den Markt bestimmten Terminkursen $r_{X}$ und $r_{Y}$ abhängig ist. (b) Bei mindestens einem verzerrten Hedge-Instrument $\left(r_{X} \neq \mathrm{E}(\tilde{r})\right.$ oder $\left.r_{Y} \neq \mathrm{E}\left(\tilde{r}_{L}\right)\right)$ gilt $\operatorname{Cov}\left(U^{\prime}\left(\tilde{W}^{*}\right), \tilde{W}^{*}\right) \neq 0$. Dies impliziert die Abhängigkeit der optimalen Risikopolitik von der Korrelation der Risiken und der Risikopräferenz des Bankmanagements. q.e.d.

Ist ein vollständiges Hedging von Refinanzierungs- und Kreditrisiken möglich, dann ist der Value at Risik 0, da die Nettozinserträge der Bank risikolos sind. Es besteht daher kein Verlustpotenzial. Ist hingegen aufgrund von nicht handelbaren (Kredit-) Risiken oder von verzerrten Preisen der Hedging-Instrumente eine vollständige Absicherung ausgeschlossen, besteht für die Bank im Optimum ein Verlustpotenzial. Das in der Bankenregulierung und Risikosteuerung zunehmend bedeutendere Konzept des Value at Risk (Johanning/Rudolph, 2000, Oehler/Unser, 2001, Broll/Wahl, 2002) gewinnt demnach bei der Risikoidentifikation und -kontrolle ein umso größeres Gewicht, je größer der Anteil der nicht handelbaren Risiken ist.

\section{Zusammenfassung}

Das Risikomanagement in Banken beinhaltet i.d.R. das Ziel, die Auswirkungen unerwünschter Marktwertänderungen auf die Ertragslage des Kreditinstituts zu neutralisieren und deren Entwicklung zu verstetigen. Eine gängige ökonomische Be- 
gründung für diese Zielsetzung der Bank besteht in der Risikoaversion des Bankmanagements. Beispielsweise besteht für die Bankmanager ein Arbeitsplatzrisiko bei Misserfolgen des Instituts.

Der Beitrag zeigt Zusammenhänge zwischen der Verfügbarkeit von HedgeInstrumenten und der Geschäftspolitik der Bank auf. Nur wenn alle Risiken marktgängig sind und die Hedge-Instrumente eine perfekte Korrelation mit der offenen Position liefern, ergeben sich relativ einfache Entscheidungsregeln für die Bankpolitik hinsichtlich des Kredit- und Einlagevolumens. Es genügt dann die Kenntnis von Marktdaten und Grenzkosten. Dies kann die Informationsbeschaffung und die bankinterne Kommunikation erleichtern. Bereits die nicht perfekte Korrelation oder Nicht-Verfügbarkeit eines Instruments der Risikoabsicherung genügt, um die optimale Entscheidung dramatisch zu erschweren. Die Geschäftspolitik der Bank hängt dann auch von Erwartungen, Risikopräferenzen, Korrelationen der Risiken und Anfangsvermögen ab. Damit existieren keine einfachen Entscheidungsregeln mehr.

\section{Literatur}

Broll, U., J. Jaenicke, Bankrisiko, Zinsmargen und flexibles Futures-Hedging, in: Schweizerische Zeitschrift für Volkswirtschaft und Statistik, Vol. 136 (2000), S. 147160.

Broll, U., J.E. Wahl, Optimum Bank Equity Capital and Value at Risk, in: C. Scholz, J. Zentes (eds), Strategic Management, Wiesbaden 2002, S. 69-82.

Burghof, H.-P., S. Henke, B. Rudolph, P.J. Schönbucher, D. Sommer (Hrsg.), Kreditderivate. Handbuch für die Bank- und Anlagepraxis, Stuttgart 2000.

Deutsche Bundesbank, Bilanzunwirksame Geschäfte deutscher Banken, Monatsbericht Oktober 1993, S. 47-63.

Deutsche Bundesbank, Das Eigenkapital der Kreditinstitute aus bankinterner und regulatorischer Sicht, Monatsbericht Januar 2002, S. 41-60.

Freixas, X., J.C. Rochet, Microeconomics of Banking, Cambridge, London 1998.

Hartmann-Wendels, Th., A. Pfingsten, M. Weber, Bankbetriebslehre, 2. Aufl., Berlin et al. 2000 .

Johanning, L., B. Rudolph (Hrsg.), Handbuch Risikomanagement, Band 1 und 2, Bad Soden/Ts. 2000.

Oehler, A., M. Unser, Finanzwirtschaftliches Risikomanagement, Berlin et al. 2001.

Pausch, Th., P. Welzel, Credit Risk and the Role of Capital Adequacy Regulation, Beitrag Nr. 224, Volkswirtschaftliche Diskussionsreihe, Universität Augsburg 2002. 
Pritsch, G., U. Hommel, Hedging im Sinne des Aktionärs - ökonomische Erklärungsansätze für das unternehmerische Risikomanagement, in: Deutsche Betriebswirtschaft, Vol. 57 (1997), S. 672-693.

Wahl, J.E., U. Broll, Financial Hedging and Banks' Assets and Liabilities Management, in: M. Frenkel, U. Hommel, M. Rudolf (eds), Risk Management: Challenge and Opportunity, Berlin et al. 2000, S. 213-227.

Wahl, J.E., U. Broll, Zur Vorteilhaftigkeit des Hedgings in Banken, in: Kredit und Kapital, Vol. 34 (2001), S. 579-589.

Wilson, Th.C., Portfolio Credit Risk, in: Federal Reserve Bank of New York Economic Policy Review, Vol. 4 (1998), S. 71-82.

Zink, H., Methoden des Kredit- und Länderrisikomanagements, in: S.K. Berninghaus, M. Braulke (Hrsg.), Beiträge zur Mikro- und Makroökonomik (Festschrift für Hans Jürgen Ramser), Berlin et al. 2001, S. 505-515. 